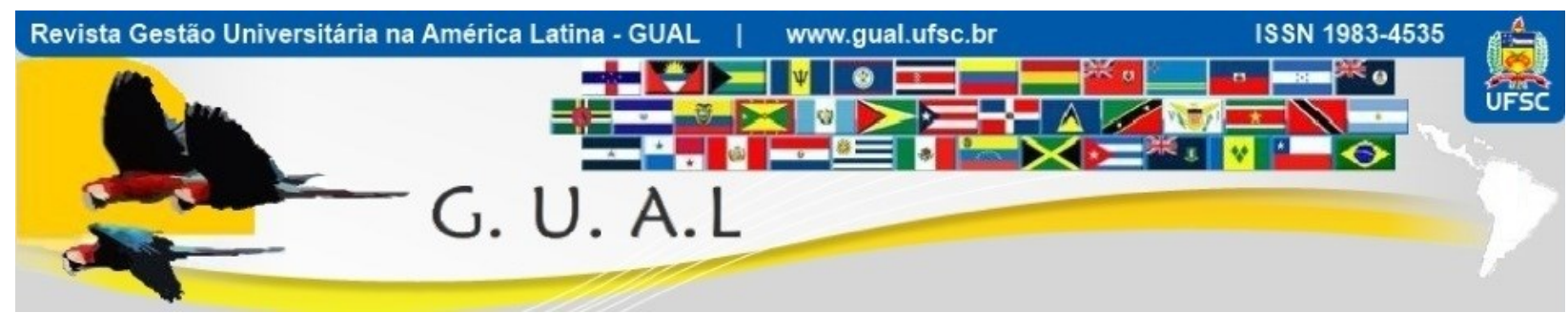

DOI: http://dx.doi.org/10.5007/1983-4535.2015v8n4p237

\title{
INDICADORES DE GESTÃO EM CONFLITO COM INDICADORES DE QUALIDADE? LIÇÕES ECONÔMICAS PARA A GESTÃO UNIVERSITÁRIA
}

\section{MANAGEMENT INDICATORS IN CONFLICT WITH QUALITY INDICATORS? ECONOMIC LESSONS TO UNIVERSITY MANAGEMENT}

Katia Maria Silva Boynard, Mestre Universidade de Brasilia - UnB katiaslva@unb.br

Jorge Madeira Nogueira, Doutor Universidade de Brasilia - UnB jmn0702@terra.com.br

Recebido em 05/dezembro/2014

Aprovado em 05/abril/2015

Sistema de Avaliação: Double Blind Review

Esta obra está sob uma Licença Creative Commons Atribuição-Uso. 


\title{
RESUMO
}

Os indicadores são instrumentos de gestão essenciais nas atividades de monitoramento e avaliação das instituições. Esta pesquisa tem como objetivo analisar a eficácia dos indicadores de gestão de uma universidade em relação aos indicadores de qualidade acadêmica, evidenciando suas correlações positivas e negativas. Numa abordagem quantitativa, utilizouse da pesquisa documental para analisar a evolução dos indicadores. Para identificar a relação entre os dois grupos, utilizou-se o teste de Coeficiente de Correlação de Pearson. Os resultados mostraram que o REUNI influenciou o desempenho dos indicadores de gestão, desempenho positivo foi verificado nos indicadores: AIPE, Conceito CAPES e IQCD. Destacou-se o expressivo aumento do CCAE e a redução da TSG. No que se refere aos indicadores de qualidade acadêmica, constatou-se que as variáveis organização didáticopedagógica e infraestrutura devem ser aprimoradas. Outro aspecto importante é a promoção efetiva da participação dos alunos no ENADE. Os testes de correlação verificaram que existe relação entre os indicadores de gestão e o Índice Geral de Cursos (IGC). Uma forte correlação foi identificada nos indicadores IQCD e Conceito CAPES.

Palavras-chave: Universidades públicas. Indicadores de gestão. Indicadores de qualidade acadêmica.

\begin{abstract}
Indicators are essential management tools for monitoring and evaluating activities at institutions. This research aims to analyze the effectiveness of university management indicators in relation to academic quality indicators, highlighting their positive and negative correlations. From a quantitative approach, a documentary research has been used so as to analyze the evolution of indicators. In order to identify the relationship between these two groups, we used the Pearson Correlation Coefficient test. The results showed that the REUNI Program has influenced performance management indicators positively on AIPE, Conceito CAPES and IQCD. As yet, the significant CCAE increase and the TSG reduction may be also highlighted. . With regard to academic quality indicators, it was found that the didacticpedagogic and infrastructure variables must be improved. Another important aspect refers to the effective students participation in the ENADE. The correlation tests identified relationship between management indicators and the General Index of Courses (IGC).As yet, a strong correlation has been identified between IQCD indicators and Conceito CAPES.
\end{abstract}

Keywords: Public universities. Management indicators. Indicators of academic quality. 


\section{INTRODUÇÃO}

Com a crescente transformação no mundo da produção e do trabalho, a formação profissional de qualidade é imprescindível para o fortalecimento da economia nacional e da maior competitividade internacional. Assim, a avaliação da educação é uma reflexão, sobre o cumprimento das finalidades, mais que dos meios. Ensino, pesquisa, extensão, aula, infraestrutura, administração, tudo isso são meios para a realização da principal função da educação superior de qualidade, a construção da cidadania e de sujeitos socialmente responsáveis (DIAS SOBRINHO, 2009).

A seleção de objetivos e métricas relacionadas é um determinante importante da eficácia de uma IES, uma vez que as decisões são tomadas com base em fatos, dados e informações quantitativas (TACHIZAWA, 2009). Atualmente no processo de avaliação das Instituições Federais de Ensino Superior (IFES) brasileiras destacam-se a avaliação da gestão e a avaliação da qualidade acadêmica.

Em consonância com o novo modelo de gestão pública, o Tribunal de Contas da União (TCU), no tocante a avaliação das IFES proferiu a Decisão n $408 / 2002$ que impõe a aferição de nove indicadores de gestão, divulgados anualmente no Relatório de Gestão das IFES e no Sistema de Informações do Ministério da Educação (SIMEC). Esta obrigatoriedade visou auxiliar o acompanhamento da gestão pelas IFES, bem como servir de parâmetro de análise para auditorias operacionais, identificando boas práticas que possam ser disseminadas e adotadas por instituições similares.

A avaliação da qualidade acadêmica é realizada por meio do Sistema Nacional de Avaliação da Educação Superior (SINAES) implantado em 2004. O SINAES está fundamentado nas avaliações institucional, de cursos e de estudantes. O conjunto dessas avaliações subsidia o cálculo de quatro indicadores de qualidade: Conceito ENADE, Conceito Preliminar de Curso (CPC), Indicador de Diferença entre os Desempenhos Observado e Esperado (IDD) e o Índice Geral de Cursos Avaliados da Instituição (IGC).

Diante do exposto sobre o atual processo de avaliação da educação superior no âmbito das IFES, o objetivo deste artigo é analisar a eficácia dos indicadores de gestão de uma universidade em relação aos indicadores de qualidade acadêmica, evidenciando suas correlações positivas e negativas. 


\section{REVISÃO DE LITERATURA}

\subsection{INDICADORES DE DESEMPENHO}

O indicador é um elemento informacional de síntese, sendo obtido a partir de um modelo de relacionamento de variáveis. Segundo Caribé (2009), o produto desse modelo, seja ele numérico ou simbólico, frente a uma escala ou parâmetros de referência, mostra a situação relativa de um determinado problema ou aspecto da realidade considerado importante ou relevante, em função do que lhe é possível estabelecer e orientar decisões, ações e atividades.

Medir é produzir informações utilizando indicadores, e fazer uso de indicadores é gerenciar com base em informações. Portanto, os indicadores devem estar normalizados, para permitir a comparabilidade, com a visualização das tendências no tempo e nos dados da própria organização, da concorrência e com os referenciais de excelência (FERNANDES, 2004; VIEIRA, 2008).

As características são usadas como critério para analisar indicadores existentes e para selecionar os melhores indicadores em um conjunto maior. Autores como Trzesniak (1998), Grateron (1999), Magalhães (2004), Brasil (2009) e TCU (2011) apontaram como características essenciais de um indicador: Validade, Comparabilidade, Estabilidade, Homogeneidade, Praticidade, Independência, Confiabilidade, Seletividade, Compreensão, Completude, Economicidade, Acessibilidade, Tempestividade, Objetividade, Portabilidade, Invariância de Escala.

Fernandes (2004) faz referência aos limites usuais dos indicadores, defende que sua elaboração deve estar fundamentada na importância dos processos e nas relações de causa e efeito observadas, de modo a oferecer uma ferramenta confiável para os gestores. A empresa deve monitorar seu uso, sua utilidade e analisar a real necessidade do indicador como instrumento de apoio na tomada de decisão, ele não deve se tornar um obstáculo ou um encargo adicional. Desta forma, será possível detectar a existência de vícios, o uso inadequado de números, que não agregam valor para a tomada de decisão, ou levam a uma decisão incorreta.

Um grave erro é importar indicadores de outras organizações, sem fazer uma análise crítica sobre sua aplicabilidade à nova realidade. Cada modelo possui vantagens e desvantagens, a adoção por um ou outro, depende de sua adequação ao contexto de características do objeto de estudo e disponibilidade de dados (CARIBÉ, 2009; FERNANDES, 2004; MAGALHÃES, 2004). 
Por fim, os indicadores são instrumentos de gestão essenciais nas atividades de monitoramento e avaliação das organizações, assim como, seus projetos, programas e políticas. O propósito principal da avaliação é a condução da instituição ao aperfeiçoamento de suas atividades, pois permite acompanhar o alcance das metas e objetivos estratégicos, o desempenho de seus produtos e processos ao longo do tempo, assim como, identificar avanços, melhorias de qualidade, correção de problemas e necessidades de mudança (VIEIRA, 2008; CARIBÉ, 2009).

\subsection{INDICADORES DE GESTÃO}

Os indicadores de gestão destinam-se a avaliar a instituição como uma entidade fornecedora de produtos e prestadora de serviços aos seus clientes atuais e potenciais, por meio da mensuração dos parâmetros estratégicos, principalmente, em seu processo de interação com o meio ambiente externo (TACHIZAWA, 2009).

Os indicadores de gestão das IFES deverão envolver o ensino, pesquisa e extensão, com o intuito de retratar o posicionamento e o impacto da sua atividade fim para a sociedade. Em consonância com a Decisão $n^{0}$ 408/2002 proferida pelo TCU, as IFES são obrigadas a divulgar anualmente em seu Relatório de Gestão e no Sistema de Informações do Ministério da Educação (SIMEC), um conjunto de nove indicadores:

1. Custo Corrente com HU / Aluno Equivalente e Custo Correntes em HU / Aluno Equivalente: destaca as despesas correntes por aluno.

2. Aluno Tempo Integral/ Professor Equivalente: mensura o número de alunos atendidos por professor.

3. Aluno Tempo Integral/Funcionário Equivalente com HU e Aluno Tempo Integral / Funcionário Equivalente sem $\mathrm{HU}$ : mostra a relação entre força de trabalho administrativa e o número de alunos.

4. Funcionário Equivalente com HU / Professor Equivalente e Funcionário Equivalente sem HU / Professor Equivalente: expressa a composição dos recursos humanos, relacionados às atividades meio e fim da instituição.

5. Grau de Participação Estudantil (GPE): indica o percentual de alunos da graduação com dedicação em tempo integral ao curso.

6. Grau de Envolvimento Discente com Pós-Graduação (GEPG): demonstra a intensidade do envolvimento discente com a pós-graduação.

7. Conceito CAPES para a Pós-Graduação: determina a qualidade dos cursos de pósgraduação, stricto sensu, avaliados pela CAPES.

8. Índice de Qualificação do Corpo Docente: evidencia o nível de qualificação dos docentes da instituição.

9. Taxa de Sucesso na Graduação (TSG): representa a relação entre o número de diplomados e número total de alunos ingressantes. 
O TCU ressalta a heterogeneidade das IFES e afirma a impossibilidade de avaliar o desempenho dessas apenas por esse conjunto de indicadores. Desta forma, os indicadores de gestão impostos pelo TCU, devem ser considerados apenas como ferramenta auxiliar no acompanhamento do desempenho, sua finalidade principal é contribuir para o aprimoramento da gestão das IFES.

\subsection{INDICADORES DE QUALIDADE ACADÊMICA}

Os indicadores de qualidade se destinam a avaliação da qualidade e de desempenho relativa a cada processo da instituição. São aqueles que refletem a relação de produtos (serviços) / insumos, ou seja, buscam medir a eficiência de um dado processo ou operação em relação à utilização de um recurso ou insumo específico (TACHIZAWA, 2009).

O Sistema Nacional de Avaliação do Ensino Superior (SINAES) foi implantado em 2004 e tem como objetivos: 1)identificar mérito e valor das instituições, áreas, cursos e programas, nas dimensões de ensino, pesquisa, extensão, gestão e formação; 2) melhorar a qualidade da educação superior, orientar a expansão da oferta e 3) promover a responsabilidade social das IES, respeitando a identidade institucional e a autonomia (INEP, 2013).

O SINAES é formado por três componentes principais: 1) avaliação das instituições, 2) avaliação dos cursos e 3) avaliação do desempenho dos estudantes. O resultado dessas três avaliações deve propiciar ao INEP, o cálculo dos quatro indicadores de qualidade do SINAES. O resultado final é formatado em valores contínuos que vão de 0 a 500, e em faixas que variam entre 1 e 5.

1. Conceito ENADE: os estudantes são avaliados pelo ENADE no início e fim do curso de graduação. $\mathrm{O}$ exame tem a periodicidade anual e é dividido em grandes áreas de conhecimento, que se repetem a cada três anos, e sua aplicação é acompanhada de instrumentos destinados ao levantamento do perfil dos estudantes. O ENADE é composto pela prova, o questionário de avaliação discente da educação, o questionário dos coordenadores de curso e a percepção do aluno sobre a prova.

2. Indicador de Diferença entre os Desempenhos Observado e Esperado (IDD): resulta da diferença entre o desempenho médio obtido no ENADE pelos estudantes concluintes de um curso e o desempenho médio que era esperado para esses mesmos estudantes, dadas as informações existentes sobre o perfil dos ingressantes desse curso (INEP, 2011a). O conceito IDD tem a função de mensurar o conhecimento agregado ao longo do curso.

3. Conceito Preliminar de Curso (CPC): criado com a finalidade de auxiliar no processo de avaliação in loco dos cursos. O CPC é uma média de diferentes medidas da qualidade 
de um curso, os três elementos são utilizados nas seguintes proporções: os insumos $(30 \%)$ o ENADE (40\%) e o IDD (30\%). A dimensão insumos é composta pela variáveis corpo docente, programa pedagógico e infraestrutura.

4. Índice Geral de Cursos Avaliados da Instituição (IGC): criado em 2008 é um indicador que pretende expressar, em um único número, a qualidade de todos os cursos de graduação, mestrado e doutorado de uma IES. O IGC é divulgado anualmente. No que se refere à graduação, é utilizado o Conceito Preliminar de Curso (CPC) e, no que se refere à pós-graduação, é utilizada a nota da CAPES.

\section{MÉTODOS E PROCEDIMENTOS}

No que se refere aos Métodos e Procedimentos, quanto ao objetivo classifica-se como uma pesquisa descritiva, pois tem a finalidade de detalhar os indicadores, sua evolução e possível relação. Quanto aos procedimentos é uma pesquisa documental, os dados foram coletados de duas fontes principais: os indicadores de gestão, extraídos do relatório de gestão das IFES e os indicadores de qualidade acadêmica, extraídos dos documentos divulgados pelo INEP. Ela busca verificar o desempenho da UnB a partir de uma análise dos dois grupos de indicadores.

Pode ser considerada uma pesquisa de ordem quantitativa, pois as informações são expostas em números a fim de que sejam analisadas. Utilizou-se da análise estatística para verificar a relação existente entre os indicadores de gestão e o indicador de qualidade acadêmica - IGC, por meio do Coeficiente de Correlação de Pearson. As correlações foram aferidas com auxílio do Software Stata versão 12.0. Essa parte da pesquisa teve como amostra 51 IFES, do universo atual de 59 IFES, em razão da ausência de dados do indicador IGC das universidades recém-criadas nos relatórios do INEP.

\section{INDICADORES NA UNB: CARACTERÍSTICAS E EVOLUÇÃO}

\subsection{EVOLUÇÃO DOS INDICADORES DE QUALIDADE ACADÊMICA}

Esta parte do estudo visa apresentar uma visão geral dos indicadores de qualidade da UnB. Os dados analisados neste item foram extraídos da base do INEP, divulgados em seu endereço eletrônico (www.inep.gov.br). Cabe ressaltar que o ENADE é aplicado por grandes áreas do conhecimento registradas no INEP, divididas em três grupos, sendo esses grupos avaliados a cada três anos. Desta maneira, a evolução dos indicadores de qualidade: Conceito Enade, IDD e CPC será demonstrada através da avaliação realizada no Grupo I, Grupo II e Grupo III, no período de 2006 a 2011. De forma contrária, o indicador IGC é divulgado anualmente pelo INEP e demonstra um resultado global para a UnB. 


\section{- GRUPO I}

O Grupo I avaliou 11 cursos, mas somente nove receberam conceitos. Os cursos de Fisioterapia e Terapia Ocupacional estão Sem Conceitos, pois são cursos novos e não possuem alunos concluintes. A Tabela 1 apresenta os indicadores Conceito Enade, IDD e CPC nos anos de 2007 e 2010 para os cursos avaliados no Grupo I.

Tabela 1 Indicadores do Grupo I

\begin{tabular}{l|c|c|c|c|c|c|c|c}
\hline \multicolumn{1}{c}{ Cursos Avaliados } & \multicolumn{9}{c|}{$\begin{array}{c}\text { Conceito } \\
\text { Enade }\end{array}$} & \multicolumn{2}{c|}{ IDD } & \multicolumn{2}{c}{$\begin{array}{c}\text { CPC } \\
\text { contínuo }\end{array}$} & \multicolumn{2}{c}{ CPC faixa } \\
\cline { 2 - 11 } & 2007 & 2010 & 2007 & 2010 & 2007 & 2010 & 2007 & 2010 \\
\hline AGRONOMIA & 5 & 4 & 1,25 & 3.38 & 3,16 & 3.68 & 4 & 4 \\
\hline EDUCAÇÃO FÍSICA & 4 & 4 & - & 3,41 & 3,95 & 3,87 & 5 & 4 \\
\hline SERVIÇO SOCIAL & 3 & 1 & 2,64 & - & 2,76 & 1,81 & 3 & 2 \\
\hline ENFERMAGEM & 4 & 1 & 2,13 & - & 3,19 & 2,20 & 4 & 3 \\
\hline FARMÁCIA & 5 & 5 & - & 2,54 & 3,85 & 3,55 & 4 & 4 \\
\hline FISIOTERAPIA & - & SC & - & - & - & - & - & SC \\
\hline MEDICINA & 4 & 4 & 3,78 & 3,25 & 3,50 & 3,40 & 4 & 4 \\
\hline MEDICINA VETERINÁRIA & 5 & 4 & 3,76 & 2,93 & 3,80 & 3,55 & 4 & 4 \\
\hline NUTRIÇÃO & 5 & 5 & 2,15 & 5,00 & 3,69 & 4,45 & 4 & 5 \\
\hline ODONTOLOGIA & 4 & 4 & 3,56 & 3,51 & 3,74 & 3,60 & 4 & 4 \\
\hline TERAPIA OCUPACIONAL & - & SC & - & - & - & - & - & SC \\
\hline
\end{tabular}

Fonte: Adaptado de INEP (2013b).

Verifica-se que o Conceito Enade, sofreu uma variação negativa no ciclo avaliado. Os cursos Agronomia e Medicina Veterinária tiveram seu conceito reduzido para 4, justificado pela média de 50 pontos nas provas de formação geral e em componentes específico. Em 2010 o conceito insatisfatório foi recebido por $22 \%$ dos cursos avaliados (Serviço Social e Enfermagem), resultado do péssimo desempenho dos alunos com uma média de apenas 10 pontos nas avaliações, conforme mostra o Relatório de Curso Enade 2010 (INEP, 2011b). Esses conceitos insatisfatórios podem ser decorrentes de um boicote dos alunos aos métodos de avaliação do ENADE.

A evolução do IDD na UnB mostra uma melhora no decorrer desse ciclo, sua média sobe de 2,75 em 2007 para 3,44 em 2010. Todos os cursos desse grupo apresentaram o indicador em nível satisfatório. Os cursos de Agronomia e Nutrição apresentam um expressivo aumento nesse indicador, sendo que a Nutrição é o único curso avaliado com o 
conceito 5. Os cursos Serviço Social e Enfermagem não apresentam o IDD 2010, devido à nota zero dos alunos ingressantes e concluintes, respectivamente.

O resultado do CPC registra bom desempenho, apresentando pouca variação. Em 2010 dos cursos avaliados na UnB 66,7\% obtiveram conceito 4. Apenas os cursos Serviço Social e Educação Física tiveram conceitos rebaixados. Não obstante, o item corpo docente seja bem avaliado, a infraestrutura requer melhoras. Destaca-se o curso de Nutrição por apresentar excelentes resultados, recebendo conceito 5 nos três indicadores apresentados. O inverso ocorre com o curso de Serviço Social, o qual merece atenção, pelo resultado insatisfatório em todos os indicadores.

\section{- GRUPO II}

O Grupo II avaliou 26 cursos. Todos os cursos de Engenharia estão concentrados nesse grupo. Os indicadores de qualidade da UnB, no período de 2008 e 2011 são demonstrados na Tabela 2.

Os resultados do Conceito Enade evidenciam desempenho inferior em $2011 \mathrm{em}$ relação a 2008. O número de cursos avaliados com o conceito máximo 5 são reduzidos: apenas três ( Ciências Sociais, Engenharia Civil e Engenharia Florestal). Entretanto o percentual dos conceitos 3 e 4 apresentam uma elevação, com destaque para os cursos Arquitetura e Geografia (bacharelado) que evoluem do conceito 2 para 4 no ciclo avaliado.

Seguindo a mesma tendência, o indicador IDD também sofre uma leve redução: a média em 2008 era 2,66 reduzindo para 2,49 em 2011. As reduções mais significativas foram observadas nos cursos: História Pedagogia, Engenharia Civil, Engenharia Mecatrônica e Engenharia Florestal. No período avaliado os cursos Arquitetura e Geografia alcançaram uma significativa progressão, ambos estavam com IDD zero em 2008 e evoluem para os índices 3,24 e 2,69 .

Ao considerar os resultados do CPC, percebe-se que o total de cursos com o conceito 5 em 2008 era 28\% declinando para apenas 5\% em 2011. A Engenharia Elétrica é o único curso a receber o conceito máximo. Em termos comparativos conseguem insatisfatório desempenho no CPC ao longo de 2011 os cursos: Engenharia Mecatrônica e Letras (licenciatura). Nesse contexto, uma análise geral do CPC no grupo II mostra que $89 \%$ dos cursos têm conceito satisfatório. Todavia, o percentual com excelência é ínfimo. 
Tabela 2 Indicadores do Grupo II

\begin{tabular}{|c|c|c|c|c|c|c|c|c|}
\hline \multicolumn{9}{|c|}{ GRUPO II } \\
\hline \multirow{2}{*}{ Cursos Avaliados } & \multicolumn{2}{|c|}{$\begin{array}{l}\text { Conceito } \\
\text { Enade }\end{array}$} & \multicolumn{2}{|c|}{ IDD } & \multicolumn{2}{|c|}{$\begin{array}{c}\text { CPC } \\
\text { contínuo }\end{array}$} & \multicolumn{2}{|c|}{ CPC faixa } \\
\hline & 2008 & 2011 & 2008 & 2011 & 2008 & 2011 & 2008 & 2011 \\
\hline $\begin{array}{l}\text { ARQUITETURA E } \\
\text { URBANISMO }\end{array}$ & 2 & 4 & 0,00 & 3,24 & 2,22 & 3,60 & 3 & 4 \\
\hline $\begin{array}{l}\text { ARTES VISUAIS } \\
\text { (LICENCIATURA) }\end{array}$ & - & 4 & - & 2,96 & - & 3,35 & - & 4 \\
\hline CIÊNCIAS SOCIAIS & 5 & 3 & 2,98 & 2,21 & 3,73 & 2,98 & 4 & 4 \\
\hline FILOSOFIA & 3 & 3 & 2,37 & 2,38 & 2,76 & 2,97 & 3 & 4 \\
\hline GEOGRAFIA & 2 & 4 & 0,00 & 2,69 & 2,17 & 2,04 & 3 & 3 \\
\hline GEOGRAFIA (LICENCIATURA) & - & 5 & - & 4,01 & - & 3,86 & - & 4 \\
\hline HISTÓRIA & 4 & 3 & 1,81 & 0,78 & 3,10 & 2,40 & 4 & 3 \\
\hline LETRAS & 4 & 3 & 1,21 & 2,41 & 3,03 & 2,55 & 4 & 3 \\
\hline LETRAS (LICENCIATURA) & - & 1 & - & 0,00 & - & 1,70 & - & 2 \\
\hline MÚSICA (LICENCIATURA) & & 3 & - & 2,57 & - & 3,07 & - & 4 \\
\hline PEDAGOGIA & 4 & 2 & 2,25 & 0,60 & 3,39 & 2,28 & 4 & 3 \\
\hline $\begin{array}{l}\text { COMPUTAÇÃO } \\
\text { (BACHARELADO) }\end{array}$ & 3 & 4 & 2,57 & 2,79 & 3,17 & 3,58 & - & - \\
\hline $\begin{array}{l}\text { COMPUTAÇÃO } \\
\text { (LICENCIATURA) }\end{array}$ & - & 5 & - & 1,73 & - & 3,45 & - & - \\
\hline ENGENHARIA CIVIL & 5 & 4 & 4,12 & 2,89 & 4,21 & 3,58 & 5 & 4 \\
\hline $\begin{array}{l}\text { ENGENHARIA DE CONTROLE } \\
\text { E AUTOMAÇÃO }\end{array}$ & 5 & - & 5,00 & - & 4,21 & - & 5 & - \\
\hline ENGENHARIA ELÉTRICA & 5 & 5 & 4,07 & 4,07 & 4,10 & 4,19 & 5 & 5 \\
\hline $\begin{array}{l}\text { ENGENHARIA DE REDES DE } \\
\text { COMUNICAÇÃO }\end{array}$ & - & 5 & - & 3,04 & - & 3,89 & - & 4 \\
\hline $\begin{array}{l}\text { ENGENHARIA } \\
\text { MECATRÔNICA }\end{array}$ & - & 3 & - & 1,47 & - & 1,57 & - & 2 \\
\hline $\begin{array}{l}\text { ENGENHARIA DE } \\
\text { TELECOMUNICAÇÕES }\end{array}$ & 5 & & 3,80 & - & 4,25 & & 5 & - \\
\hline ENGENHARIA MECÂNICA & 4 & 4 & 2,88 & 3,23 & 3,43 & 3,83 & 4 & 4 \\
\hline ENGENHARIA FLORESTAL & 5 & 4 & 5,00 & 3,27 & 4,42 & 3,43 & 5 & 4 \\
\hline FÍSICA & 3 & 3 & 2,31 & 2,51 & 3,21 & 3,22 & 4 & 4 \\
\hline MATEMÁTICA & 4 & 4 & 2,60 & 2,12 & 3,52 & 3,21 & 4 & 4 \\
\hline QUÍMICA & 3 & 3 & 2,30 & 2,27 & 3,30 & 3,18 & 4 & 4 \\
\hline BIOLOGIA (BACHARELADO) & 5 & 5 & 2,59 & 3,13 & 3,64 & 3,85 & 4 & 4 \\
\hline BIOLOGIA (LICENCIATURA) & - & 4 & - & 3,27 & - & 3,84 & 4 & - \\
\hline
\end{tabular}

Fonte: Adaptado de INEP (2013b). 


\section{- GRUPO II}

O Grupo III avaliou 14 cursos, em sua maioria na área de humanas e o ciclo de avaliação compreendeu os anos de 2006 e 2009. Ressalta-se que as informações referentes ao ano de 2012, até o momento da coleta, ainda não haviam sido divulgadas pelo INEP. O ano de 2006 não apresenta o indicador CPC, pois esse foi criado apenas em 2008. A Tabela 3 apresenta o conjunto de indicadores de qualidade avaliados no Grupo III.

Tabela 3 Indicadores do Grupo III

\begin{tabular}{|c|c|c|c|c|c|c|c|c|}
\hline \multicolumn{9}{|c|}{ GRUPO III } \\
\hline \multirow[t]{2}{*}{ Cursos Avaliados } & \multicolumn{2}{|c|}{\begin{tabular}{|c|} 
Conceito \\
Enade
\end{tabular}} & \multicolumn{2}{|c|}{ IDD } & \multicolumn{2}{|c|}{$\begin{array}{c}\text { CPC } \\
\text { contínuo }\end{array}$} & \multicolumn{2}{|c|}{ CPC faixa } \\
\hline & 2006 & 2009 & 2006 & 2009 & 2006 & 2009 & 2006 & 2009 \\
\hline ADMINISTRAÇÃO & 5 & 5 & 4,00 & 3,62 & - & 4,19 & - & 5 \\
\hline ARQUIVOLOGIA & 3 & 4 & 5,00 & $\mathrm{SC}$ & - & 3,82 & - & 4 \\
\hline BIBLIOTECONOMIA & 4 & 4 & 5,00 & 5,00 & - & 3,87 & - & 4 \\
\hline CIÊNCIAS CONTÁBEIS & 5 & 5 & 5,00 & 3,61 & - & 4,30 & - & 5 \\
\hline CIENCIAS ECONOMICAS & 5 & 5 & 5,00 & 5,00 & - & 4,34 & - & 5 \\
\hline $\begin{array}{ll}\text { COMUNICAÇÃO } & \text { SOCIAL } \\
\text { JORNALISMO } & \\
\end{array}$ & 4 & 3 & 5,00 & 0,86 & - & 2,66 & - & 3 \\
\hline $\begin{array}{lcl}\text { COMUNICAÇÃO } & \text { SOCIAL } & - \\
\text { PUBLICIDADE E PROPAGANDA } & \end{array}$ & 4 & 3 & 5,00 & 1,56 & - & 2,82 & - & 3 \\
\hline DESIGN & 4 & 5 & 3,00 & 3,81 & - & 4,11 & - & 4 \\
\hline DIREITO & 5 & 5 & 4,00 & 2,62 & - & 3,61 & - & 4 \\
\hline MÚSICA & 5 & 3 & 4,00 & 2,37 & - & 2,97 & - & 4 \\
\hline RELAÇÕES INTERNACIONAIS & - & 5 & - & 3,16 & - & 3,73 & - & 4 \\
\hline TEATRO & 2 & 3 & 5,00 & 2,37 & - & 2,77 & - & 3 \\
\hline ESTATÍSTICA & - & 4 & - & $\mathrm{SC}$ & - & 3,39 & - & 4 \\
\hline PSICOLOGIA & 5 & 5 & 3,00 & 3,27 & - & 3,92 & - & 4 \\
\hline
\end{tabular}

Fonte: Adaptado de INEP (2013b).

O Conceito Enade mostra um desempenho superior em 2009. Como pode ser observado todos os cursos avaliados estão nível satisfatório e o conceito 5 permanece em equilíbrio $(50 \%)$. Apenas três cursos tiveram seus conceitos rebaixados: Comunicação (Jornalismo e Publicidade) e Música.

Em relação ao IDD, ocorre uma redução da excelente média 4,42 em 2006 para 3,50 em 2009. Ainda assim, é a melhor média do IDD entre os três grupos analisados. Na UnB o destaque em 2009 no indicador IDD é para os cursos de Biblioteconomia e Ciências Econômicas, ambos com o conceito 5, decorrente do processo de agregação de conhecimentos ao longo da trajetória dos estudantes nesses cursos. 
Dos 14 cursos que apresentam CPC em 2009, todos estão em nível satisfatório. O conceito 4 representa $57 \%$ dos cursos. Já o conceito mediano três pertence aos cursos: Comunicação (Jornalismo e Publicidade) e Teatro. Os cursos Administração, Ciências Contábeis e Ciências Econômicas, compõem os $22 \%$ que receberam conceito 5 , e se tornam referência para a região. O destaque no Grupo III é o curso Ciências Econômicas, que alcança o conceito máximo em todos os indicadores analisados.

\section{- Índice Geral de Cursos Avaliados da Instituição (IGC)}

O IGC é um Indicador que busca expressar a qualidade de todos os cursos de Graduação, Mestrado e Doutorado de uma Instituição. O Gráfico 1 mostra que esse indicador permaneceu estável na UnB. Nos cincos anos analisados a universidade permanece com o conceito 4, mas registrando pequenas oscilações. O ano de 2010 apresenta o melhor nível desse indicador $(3,91)$ se aproximando do conceito máximo 5 que é obtido com notas acima de 3,945. Contudo em 2011 sofre um decréscimo de 0,03.

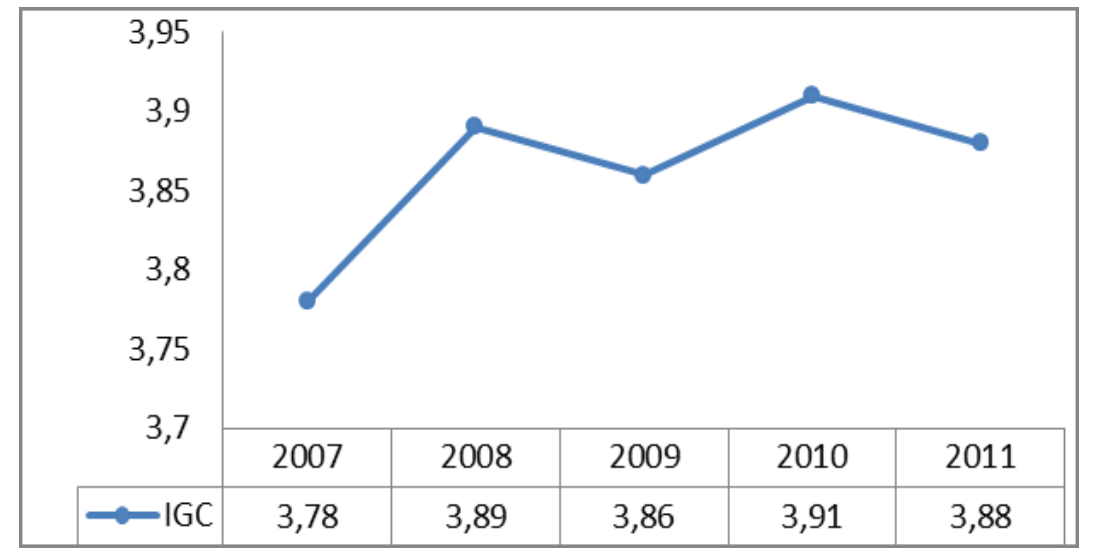

Gráfico 1 Índice Geral de Cursos.

Fonte: Elaboração própria.

Os dados apresentados para o IGC sugerem uma melhoria na qualidade dos cursos de graduação e pós-graduação da UnB, já que este índice reflete o desempenho dos estudantes e o oferecimento de infraestrutura e organização didático-pedagógica. Cabe ressaltar que ainda serão refletidos nesse indicador os impactos causados pelos investimentos do REUNI, devido ao IGC ser calculado levando-se em conta sempre o último triênio explicando dessa forma, uma defasagem na qualidade dos cursos apresentada. 


\subsection{Evolução dos indicadores de gestão}

Nesta seção apresenta-se o conjunto de indicadores de gestão da UnB definidos pelo TCU, a fim de se estabelecerem parâmetros adequados à avaliação sobre o efetivo desempenho da gestão. Com o intuito de evidenciar a evolução, optou-se por apresentar separadamente os nove indicadores. Nas demonstrações gráficas além dos resultados da UnB, visualiza-se a média nacional das IFES brasileiras, divulgadas pela SESu/MEC também em seu Relatório de Gestão.

\section{1) Custo Corrente / Aluno Equivalente}

O Indicador Custo Corrente /Aluno Equivalente busca retratar a forma como os recursos alocados na produção de ensino e pesquisa estão sendo utilizados. Analisando o Gráfico 2 verifica-se uma elevação de 56\%, justificada pela implantação do Programa REUNI (Programa de Apoio a Planos de Reestruturação e Expansão das Universidades Federais), que disponibilizou um montante superior de recursos para as IFES. Outro fator que contribuiu para o aumento dos custos foi a recomposição salarial dos servidores das IFES realizada pelo Governo Federal durante o período. Os dados mostram o indicador 93\% acima da média nacional. Cabe ressaltar, que ainda não foram estabelecidos padrões que determinem, de acordo, com o orçamento destinado pelo MEC anualmente às IFES, o custo ideal de um aluno, devido às realidades distintas e heterogêneas.

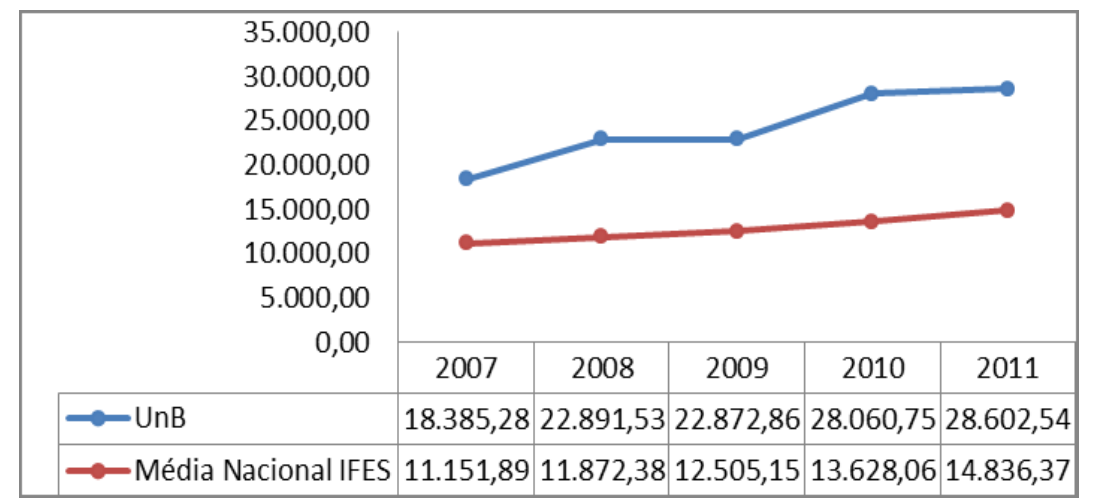

Gráfico 2 Custo Corrente /Aluno Equivalente Fonte: Elaboração própria.

\section{2) Aluno Tempo Integral / Professor Equivalente}

O indicador Aluno Tempo Integral/Professor Equivalente objetiva mensurar a produtividade do corpo docente, a partir do número de alunos atendidos por professor 
equivalente. O Gráfico 3 demonstra que inicialmente o AIPE tem uma discreta elevação até 2008, a partir desse período as ações do Reuni passa a influenciar o indicador.

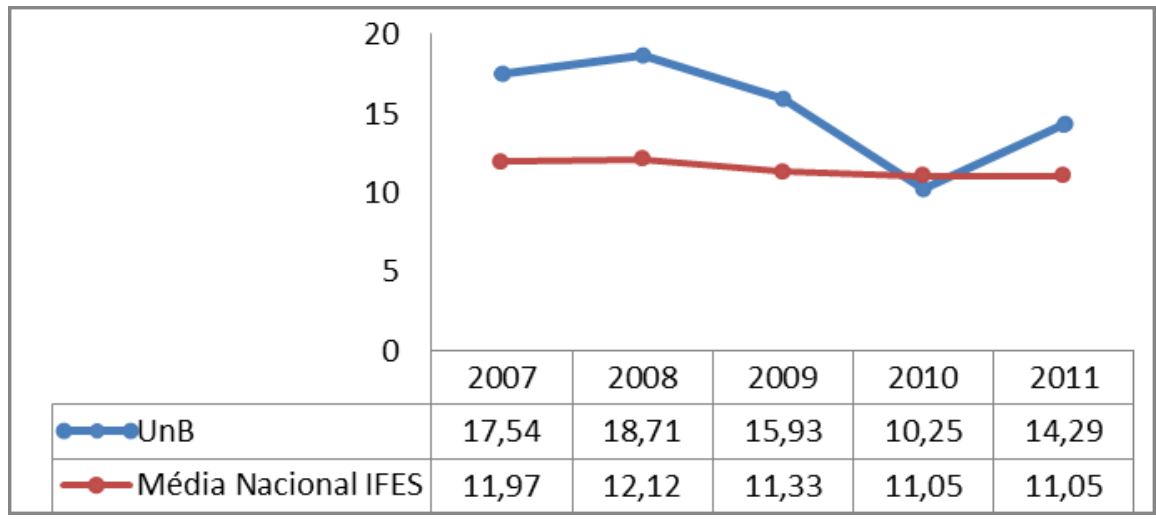

Gráfico 3 Aluno Tempo Integral / Professor Equivalente.

Fonte: Elaboração própria.

A notória queda em 2010 é decorrente do aumento (9\%) no quadro docente em proporção maior que o aumento dos alunos da graduação (6\%). Fato semelhante, mas em sentido inverso, ocorre em 2011, embora o número de professores continue a aumentar, há uma expressiva elevação no número de alunos em tempo integral. Destaca-se que o número de alunos na residência médica, praticamente dobrou e o percentual de alunos na pósgraduação subiu 20\%, devido a criação de 4 cursos de Mestrado Acadêmico e 4 de Doutorado em 2010 .

\section{3) Aluno Tempo Integral / Funcionário Equivalente}

O indicador Aluno Tempo Integral/Funcionário Equivalente avalia a produtividade do servidor equivalente da $\mathrm{UnB}$, em relação ao número de alunos em tempo integral. Como pode ser observado no Gráfico 4 o AIFE apresenta queda no período de 2009 a 2011. Embora a efetiva expansão da UnB tenha propiciado a elevação em $25 \%$ dos alunos em tempo integral, o aumento no número de funcionário equivalente duplicou na série analisada. $O$ Reuni atendendo a demanda das universidades possibilitou a contratação de técnico-administrativos, mas essa expressiva elevação é decorrente da inclusão no cálculo desse indicador de 3.057 funcionários de empresas terceirizadas. Nesse total, estão incluídos 574 terceirizados do hospital universitário (FUB, 2012a). 


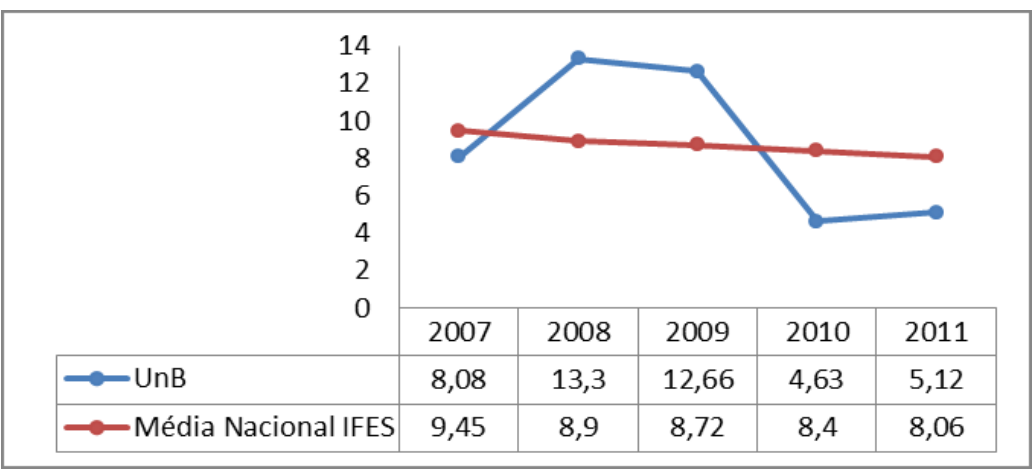

Gráfico 4 Aluno Tempo Integral / Funcionário Equivalente. Fonte: Elaboração própria.

\section{4) Funcionário Equivalente / Professor Equivalente}

O indicador de eficiência Funcionário Equivalente/Professor Equivalente, quantifica a composição dos recursos humanos, relacionados às atividades meio e fim da instituição. $\mathrm{O}$ Gráfico 5 mostra a elevação do FEPE na série histórica. O indicador exibe redução nos anos 2008 e 2009, o menor resultado da série foi 1,26 funcionários para cada professor, esse desempenho foi motivado pelo aumento de professores equivalentes. A significativa elevação de $122 \%$ a partir de 2009 resulta da inserção de 3.057 funcionários terceirizados, no componente "Funcionário Equivalente", identificada anteriormente na análise do indicador AIFE. Nesse contexto a UnB no indicador Funcionário Equivalente/Professor Equivalente se afasta da média nacional em $85 \%$.

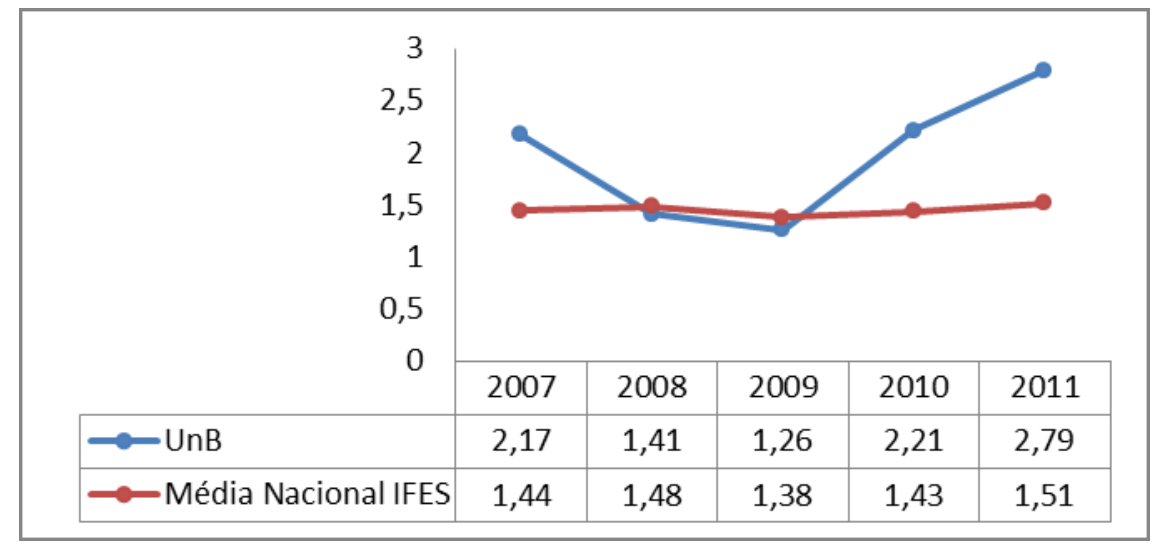

Gráfico 5 Funcionário Equivalente / Professor Equivalente.

Fonte: Elaboração própria.

\section{5) Grau de Participação Estudantil (GPE)}

O Grau de Participação Estudantil objetiva quantificar a intensidade de utilização da capacidade instalada da Universidade pelos alunos e a velocidade de integralização curricular. 
Esse indicador representa a relação entre o Aluno Tempo Integral da graduação pelo total de alunos matriculados nos cursos de graduação. Podemos aferir, com base no Gráfico 6 que o GPE apresentou melhor desempenho no ano de 2008 atingindo o resultado de 0,95.

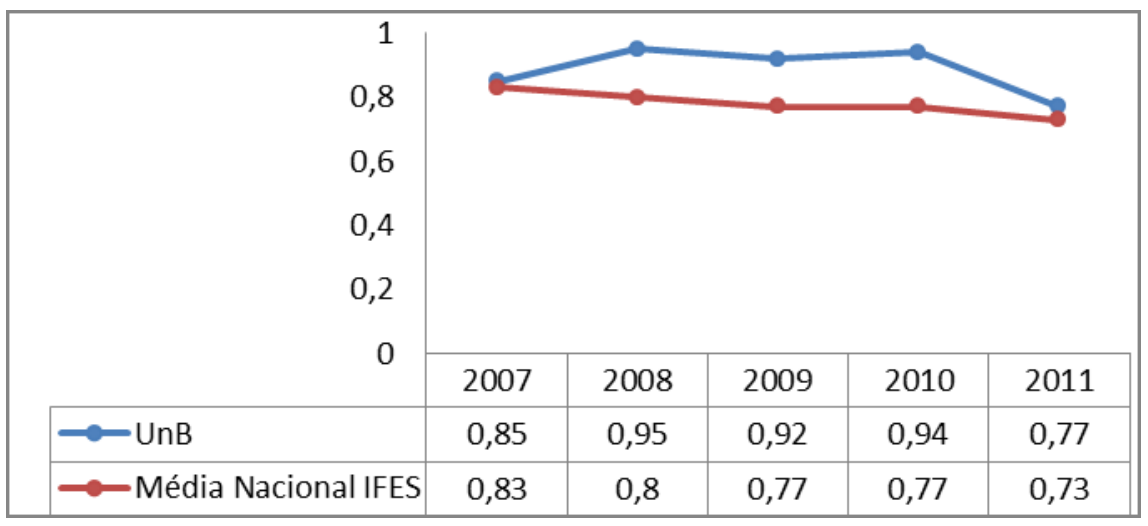

Gráfico 6 Grau de Participação Estudantil.

Fonte: Elaboração própria.

O indicador segue apresentando pequenas oscilações até uma queda significativa em 2011, resultado da redução do número de alunos da graduação em tempo integral. Destaca-se que o Reuni incentivou o aumento de vagas em cursos noturnos, em 2010 foram criados 7 cursos noturnos, o que justifica a redução do GPE. A UnB no indicador GPE apresentou resultados em sua série histórica acima da média nacional, até mesmo em 2011 com a expressiva redução explicada anteriormente.

\section{6) Grau de Envolvimento Discente com Pós-Graduação (GEPG)}

O Grau de Envolvimento Discente com a Pós-Graduação tem como objetivo retratar o grau de envolvimento em atividades de pesquisa e pós-graduação, expressando-se através da relação entre o número de estudantes vinculados a pós-graduação e o total de estudantes matriculados. No Gráfico 7, observa-se que não houve variação significativa nos resultados deste indicador. O pequeno decréscimo registrado em 2011, redução para 0,17 é decorrente do crescimento da oferta de vagas de graduação, de modo que quanto maior a expansão da graduação em relação à pós-graduação o indicador tende a diminuir. No longo prazo, a implementação de iniciativas específicas de expansão da pós-graduação poderá encadear outro movimento coordenado e sustentável de elevação desse indicador. 


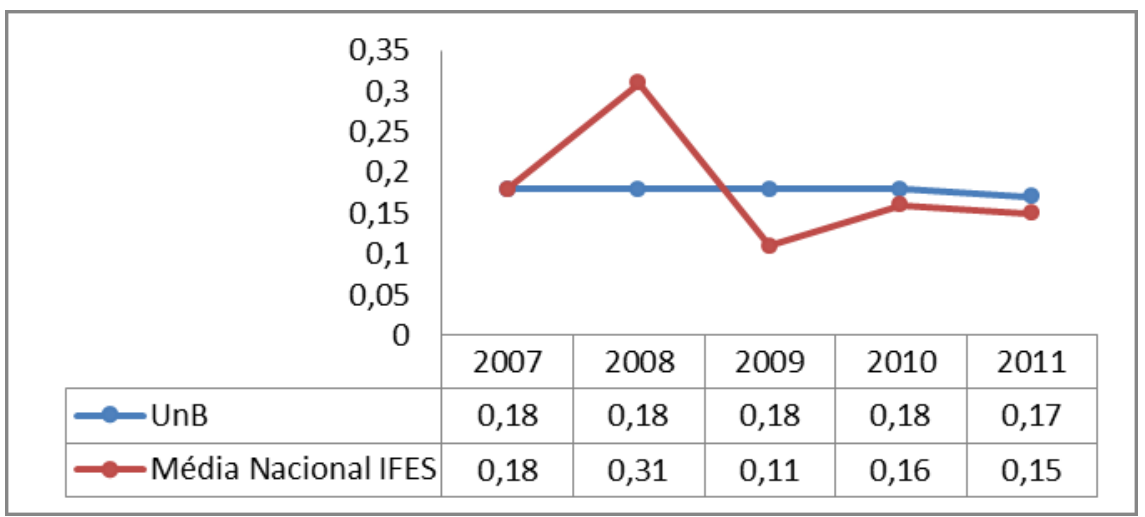

Gráfico 7 Grau de Envolvimento Discente com Pós-Graduação. Fonte: Elaboração própria.

\section{7) Conceito CAPES}

A evolução do indicador Conceito CAPES expressa a melhoria continuada nos níveis de qualidade dos cursos de pós-graduação. O Gráfico 8 expõe que no período analisado esse indicador teve relativa melhora em 2011, ampliando-se para 4,34. Salienta-se que dos 142 cursos de pós-graduação oferecidos, 22 têm nota de 5 a 7; ou seja, são bem avaliados. Outros 120 têm notas no intervalo de 3 a 4, sendo que a maioria dos cursos com nota 3, foram criados recentemente. Ao analisar a evolução do Conceito CAPES, percebe-se que a UnB em todos os anos, se afasta da média nacional positivamente.

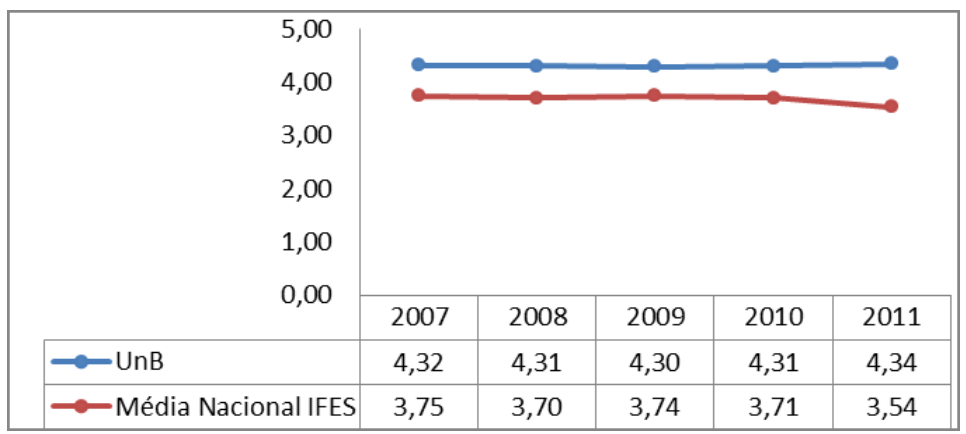

Gráfico 8 Conceito CAPES.

Fonte: Elaboração própria.

\section{8) Índice de Qualificação do Corpo Docente (IQCD)}

O Índice de qualificação do corpo docente objetiva mensurar a qualificação do corpo docente da instituição fundamentada na titulação. A pontuação máxima do IQCD é 5 . Ao observar o Gráfico 9 nota-se que a série histórica já se inicia com um bom índice $(4,26)$ em 2007, a tendência de alta permanece, evoluindo 6\% até 2011. 


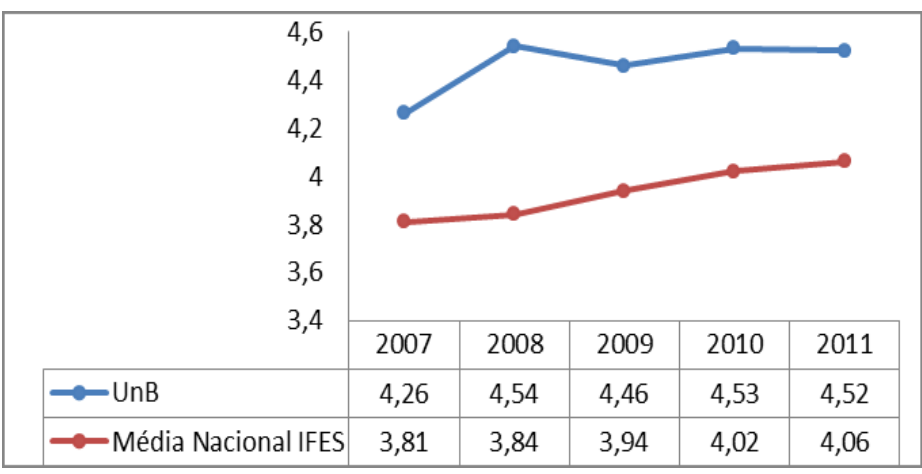

Gráfico 9 Índice de Qualificação do Corpo Docente.

Fonte: Elaboração própria.

Enfatiza-se que o significativo IQCD de 4,5 e percentual de doutores de $79 \%$, é resultado da predominância nos concursos para docentes da exigência de titulação mínima em nível de doutorado, assim como, da política institucional de incentivo à qualificação docente.

\section{9)Taxa de Sucesso na Graduação (TSG)}

A Taxa de Sucesso na Graduação é resultante da razão entre o número de diplomados e o número de ingressantes, ajustados pelo ano em que esses alunos ingressaram na UnB e o tempo de permanência fixado pela SESu/MEC para cada curso. A UnB tinha taxa superior a 70\%, conforme expressa o Gráfico 10. Em 2011 decresce 20\%, passando a TSG para 60,67\%. Cabe ressaltar que o Programa REUNI tem como meta uma taxa de sucesso na graduação de 90\%. A redução da TSG está relacionada com a evasão e retenção discente.

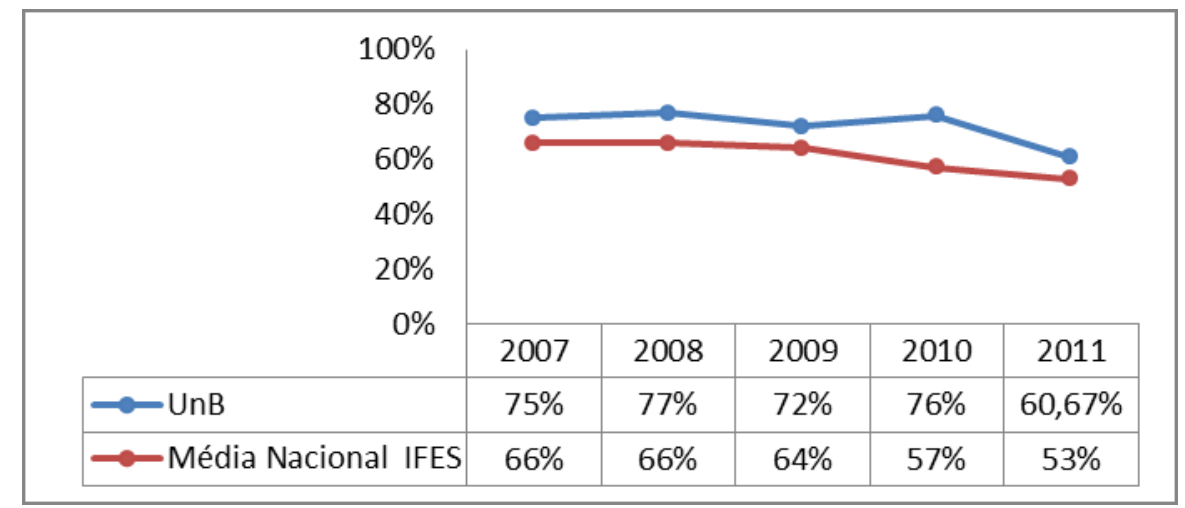

Gráfico 10 Taxa de Sucesso na Graduação.

Fonte: Elaboração própria.

Algumas falhas foram percebidas no decorrer do estudo desse grupo de indicadores: os Relatórios de Gestão da UnB não apresentam análise dos resultados dos indicadores, não constam relatos sobre as ações adotadas, para que possíveis disfunções venham a ser 
ajustadas, ou oportunidades futuras aproveitadas. Também foram identificadas divergências de informações, provavelmente relacionadas à coleta de dados. Outra falha refere-se à metodologia de cálculo dos indicadores GPE e GPEG, que não expressam a real participação dos alunos na graduação e pós-graduação.

\subsection{Correlação entre os indicadores de gestão e os indicadores de qualidade acadêmica}

Essa seção destina-se a verificar se existe correlação entre os dois grupos de indicadores estudados. Foram selecionadas como variáveis, os resultados dos indicadores de gestão e do indicador de qualidade acadêmica: Índice Geral de Cursos Avaliados da Instituição (IGC), todos divulgados anualmente. A escolha do indicador IGC justifica-se por ser uma média ponderada dos conceitos dos cursos de graduação e de pós-graduação stricto sensu da Instituição. O IGC agrega informações dos outros indicadores de qualidade estudados e expressa a qualidade do ensino superior ofertado pela UnB.

Os coeficientes de correlação tratam da quantificação do grau de dependência entre duas variáveis. Para esta análise foi utilizado o método estatístico do Coeficiente de Correlação de Pearson (r), que é uma medida de associação linear entre variáveis. O resultado apresentará um valor no intervalo de -1 até +1 . O sinal indica direção positiva ou negativa do relacionamento e o valor sugere a força da relação entre as variáveis. Quanto mais próximo de +1 ou -1 mais forte é a correlação dos dados; e quanto mais próximo de 0 (zero), a correlação é mais fraca, sendo que +1 é a correlação positiva perfeita e -1 a correlação negativa perfeita.

A amostra para a verificação dos níveis de correlação foi composta por 51 universidades federais, do universo atual de 59 IFES, em razão da ausência de dados do indicador IGC das universidades recém-criadas nos relatórios do INEP. Os dados foram processados com auxílio do Software Stata versão 12.0. A Tabela 4 apresenta o nível de correlação entre os indicadores de gestão e o indicador de qualidade acadêmica - IGC.

Ao verificar a correlação entre os indicadores de gestão e os resultados alcançados pelas IFES no Índice Geral de Cursos Avaliados da Instituição (IGC), os testes mostraram que dos nove indicadores analisados apenas o Índice de Qualificação do Corpo Docente $(r=0,81)$ e o Conceito CAPES $(r=0,74)$ apresentam forte correlação, justificada pela metodologia de cálculo do IGC, que é uma média ponderada dos conceitos dos cursos de graduação e de pósgraduação. Para a avaliação da pós-graduação é realizada uma conversão das notas fixadas pela CAPES e para a graduação é considerado o indicador Conceito Preliminar de Curso 
(CPC), que contêm em sua fórmula a variável corpo docente. Uma correlação de nível moderado foi identificada na Taxa de Sucesso na Graduação $(r=0,51)$.

Tabela 4 Correlação entre os indicadores de gestão e o IGC.

\begin{tabular}{l|c|c|c|c|c}
\hline & $\mathbf{2 0 0 7}$ & $\mathbf{2 0 0 8}$ & $\mathbf{2 0 0 9}$ & $\mathbf{2 0 1 0}$ & $\mathbf{2 0 1 1}$ \\
\hline CCAE & 0.31 & 0.29 & 0.27 & 0.26 & 0.24 \\
\hline AIPE & 0.27 & 0.40 & 0.37 & 0.34 & 0.34 \\
\hline AIFE & $-0,06$ & 0.06 & $-0,04$ & $-0,13$ & $-0,09$ \\
\hline FEPE & 0.21 & 0.19 & 0.27 & 0.31 & 0.35 \\
\hline GPE & 0.46 & 0.51 & 0.50 & 0.52 & 0.36 \\
\hline GPEG & $-0,06$ & $-0,06$ & $-0,10$ & $-0,15$ & $-0,17$ \\
\hline CAPES & 0.62 & 0.74 & 0.74 & 0.78 & 0.74 \\
\hline IQCD & 0.62 & 0.74 & 0.74 & 0.85 & 0.81 \\
\hline TSG & 0.25 & 0.24 & 0.53 & 0.51 & 0.51 \\
\hline
\end{tabular}

Fonte: Elaboração própria.

Os indicadores CCAE, AIPE, GPE e FEPE possuem fraca correlação com o resultado obtido no IGC. Ao contrário do que teoricamente se esperava os indicadores Aluno Tempo Integral / Funcionário Equivalente (AIFE) e Grau de Envolvimento Discente com PósGraduação (GPEG) apresentam correlação negativa e não se relacionam com o desempenho no IGC.

\section{CONCLUSÃO}

O objetivo da pesquisa foi analisar a eficácia dos indicadores de gestão de uma universidade em relação aos indicadores de qualidade acadêmica, evidenciando suas correlações positivas e negativas. Para isso, utilizou-se da pesquisa documental para compreender e analisar a evolução de cada um dos indicadores estudados.

No que se refere à avaliação dos indicadores de gestão, os resultados apontaram a influência do REUNI na série histórica analisada. O desempenho positivo foi verificado nos indicadores: AIPE, Conceito CAPES e IQCD. Constatou-se que a abrupta inclusão de servidores terceirizados no componente primário Funcionário Equivalente, distorceu os resultados dos indicadores AIFE e FEPE, disfarçando a insuficiência de servidores efetivos. Também foram percebidas falhas metodológicas nos indicadores GPE e GPEG, assim como, na coleta de dados pelas IFES. A principal constatação entre os indicadores de gestão foi o expressivo aumento do Custo Corrente /Aluno Equivalente (CCAE), simultaneamente à 
redução da TSG, o que confirma que maiores gastos não são suficientes para melhores resultados no sucesso da formação discente.

Por sua vez, a avaliação do conjunto de indicadores de qualidade acadêmica, não apresentou um significativo desempenho no período analisado. Entre os itens avaliados, as variáveis organização didático-pedagógica e infraestrutura, merecem atenção e devem ser aprimoradas. Verificou-se que as universidades precisam preparar e estimular seus alunos à participação na prova ENADE, ressaltando sua importância para os indicadores de qualidade da instituição.

Os resultados dos testes de correlação mostraram que há relação entre os indicadores de gestão e o Índice Geral de Cursos Avaliados da Instituição (IGC), indicador que expressa qualidade do ensino oferecido pelas IFES. Constatou-se que os indicadores IQCD e Conceito CAPES possuem forte relação com o IGC, já a TSG demonstrou um coeficiente de correlação de nível moderado.

O estudo demonstrou que as informações obtidas, permitem identificar tanto os pontos fortes como os menos desenvolvidos, funcionando como uma forma de conhecimento sobre a própria instituição, o que possibilita o aprimoramento das práticas de gestão universitária. Os resultados também são relevantes aos órgãos governamentais, para orientar suas decisões quanto as políticas públicas direcionadas à educação superior, no que ser refere à avaliação da qualidade do ensino ofertado e a accountability dos recursos disponibilizados.

\section{REFERÊNCIAS}

BRASIL. Ministério do Planejamento, Orçamento e Gestão - Secretaria de Gestão. Guia Referencial para Medição de Desempenho e Manual para Construção de Indicadores. Brasília: MP, 2009. 112 p.

CARIBÉ, Rita de Cássia do Vale. Sistema de indicadores: uma introdução. Revista Digital de Biblioteconomia e Ciência da Informação, v. 6, n. 2, p.1-23, jan./jun. 2009.

DIAS SOBRINHO, José. Avaliação Institucional para Re-institucionalizar a Educação como Bem Público. QUAESTIO: Revista de Estudos em Educação, v. 11, n. 2, p. 17-25, 2009.

FERNANDES, Djair Roberto. Uma contribuição sobre a construção de indicadores e sua importância para a gestão empresarial. Revista da FAE, Curitiba, v.7, n.1, p.1-18, jan./jun.2004.

FUB. FUNDAÇÃO UNIVERSIDADE DE BRASÍLIA. Relatório Anual de Gestão 2012. Brasília, 2013. Relatório Anual de Gestão 2011. Brasília, 2012a 
GRATERON, Ivan Ricardo Guevara. Auditoria de gestão: utilização de indicadores de gestão no setor público. Caderno de Estudos, São Paulo, n. 21, p.1-18, ago. 1999.

INEP. Instituto Nacional de Estudos e Pesquisas Educacionais Anísio Teixeira. ENADE 2010

- Relatório de Curso Serviço Social. Brasília, 2011b. Disponível em:

$<$ http://enadeies.inep.gov.br/enadeIes/enadeResultado/>. Acesso em: 10 fev. 2013.

Planilhas Resultados do ENADE. Brasília, 2013b. Disponível em:

$<$ http://portal.inep.gov.br/planilhas-enade>. Acesso em: 11 fev. 2013.

MAGALHÃES, Marcos Thadeu Queiroz. (2004). Metodologia para Desenvolvimento de Sistemas de Indicadores: Uma Aplicação no Planejamento e Gestão da Política Nacional de Transportes. 2004. 135f. Dissertação (Mestrado em Engenharia Civil e Ambiental) -

Universidade de Brasília, Brasília, DF, 2004.

TACHIZAWA, Takeshy. Princípios de produtividade e qualidade aplicados a instituições de educação superior. Revista Brasileira de Docência, Ensino e Pesquisa em Administração, v. 1, n. 1, p.33-51, maio 2009.

TCU. Tribunal de Contas da União. Decisão no 408/2002. Plenário. Auditoria Operacional. Relatório consolidado. Universidades federais. UNB. FUAM. UFGO. UFPE. UFRJ. UFRS. Elaboração de diagnóstico na área de ensino público superior. Levantamento de indicadores de desempenho. Determinação. Ciência ao Congresso Nacional e órgãos de supervisão e controle. Juntada às respectivas contas. . Brasília, 2002.

.Técnica de indicadores de desempenho para auditorias. Secretaria de Fiscalização e Avaliação de Programas de Governo (Seprog), 2011. 37 p.

TRZESNIAK, Piotr. Indicadores quantitativos: reflexões que antecedem seu estabelecimento. Ciência da Informação, Brasília, v. 2, n. 2, p.159-164, maio/ago. 1998.

VIEIRA, Edzana Roberta Ferreira da Cunha. Características da medição de desempenho organizacional: um estudo descritivo nos hospitais do estado do Rio Grande do Norte. 2008. 107f. Dissertação (Mestrado em Ciências Contábeis) -Programa Multiinstitucional e Inter-Regional de Pós- Graduação em Ciências Contábeis, Universidade de Brasília/UFPB/UFPE/UFRN, Natal, RN, 2008. 